\title{
Completely mechanical quick changeable joints for multipurpose explosive ordnance disposal robots E. Faruk Kececi
}

\author{
Department of Mechanical Engineering, Izmir Institute of Technology, Urla, Izmir 35430, Turkey. \\ E-mail:farukkececi@iyte.edu.tr
}

(Received in Final Form: July 7, 2008. First published online: August 14, 2008)

\begin{abstract}
SUMMARY
This paper is an investigation of completely mechanical quick changeable joints for multipurpose explosive ordnance disposal (EOD) robots. With the assistance of a quick changeable joint, an ordinary EOD robot becomes a multipurpose robot with an end effector which can be switched during the task. This exchangeable end effector permits the robot to perform more complex duties. Making the joint completely mechanical increases its capacity and decreases its complexity of control and risk of failure. In this paper, the design, manufacturing, and testing stages are explained for four quick changeable joints each possessing different physical working principles. The test results reveal the best design for a multipurpose EOD robot and give ideas for other uses of quick changeable joints. Employing the quick changeable joints in other mobile robot applications can increase a robot's capacity and efficiency.
\end{abstract}

KEYWORDS: Quick changeable joint; Multipurpose robot; Joint testing.

\section{Introduction}

The aim of this research was to design completely mechanical quick changeable joints for multipurpose explosive ordnance disposal (EOD) robots which are designed to disarm explosive ordnance remains. Quick changeable joints allow these robots to change their end effectors autonomously. This increases the robot's capacity in handling various shaped objects and completing different tasks by changing the tools in a short period of time without being called back to the control station.

The quick changeable joint is a device that when attached to the wrist of a robotic arm, Fig. 1, provides the robot with the ability to automatically change its end effectors when necessary. This device will increase a robot's capacity in handling various shaped objects and improve its adaptability to different EOD tasks.

EOD robots either carry an explosive ordnance into a containment vessel to be disposed or when the explosive material cannot be moved its triggering mechanism is separated to stop the explosion. With an EOD robot, the disarming of an explosive ordnance can be achieved remotely.

Current EOD robots only have a standard gripper. ${ }^{1,2}$ This gripper is insufficient for the disposal of explosives because it can only carry the explosive objects, it cannot disarm the triggering mechanism. In order to neutralize an explosive object, a robot needs some special manipulators such as cutting, screwing, precise gripping, internal clamping, or drilling tools. Figure 2 shows the different-purpose tools of EOD robot.

In a disarming operation, it is hazardous for an operator to approach the robot and switch the end effector by hand. An autonomous quick changing mechanism is needed to change the onboard tools. An autonomous system for this mechanism is desired in order to circumvent a return to the base and therefore would eliminate waiting for the operator to complete a switch of the end effector. Returning to the base consumes too much time and it could lead to the explosion of time-based bombs.

A similar connection mechanism exists in computer numeric controlled $(\mathrm{CNC})$ machines to change the cutting tool powered by a pneumatic system. ${ }^{3}$ Even pneumatic systems are used in industrial robotic application ${ }^{4,5}$; however, since this system requires a compressor and a pressure tank, it is not easily applicable in a mobile robot application.

Different gripper designs have been studied and applied to industry very successfully ${ }^{6-8}$; however, without a quick joint mechanism, an EOD robot cannot use different grippers without returning to the base.

If all the different end effectors were placed at the end of the robot arm, the robot arm would become too bulky and too heavy requiring more powerful actuators and battery. ${ }^{4}$ The proposed solution is to place the end effectors on the robot body so that the end effector can be replaced quickly and autonomously with the help of the quick change joint.

The different commercially available EOD robots are examined and the end effector type and their changeability are investigated if they exist. These studies proved that the commercial EOD robots do not have a quick change joint. The EOD robot systems are presented to the market with different arm and gripper designs having 6-7-degreesof-freedom (DOF). Considering the range of tasks to be accomplished during an operation, one type of end effector design is insufficient; so, two or more alternative end effectors are presented by the manufacturers. However, in current robots crucial time is lost because end effectors can only be switched by human controllers and in order to do this safely the robot must retreat a safe distance from the explosive ordnance. An autonomous change can only be achieved by an automatic quick changing joint. 


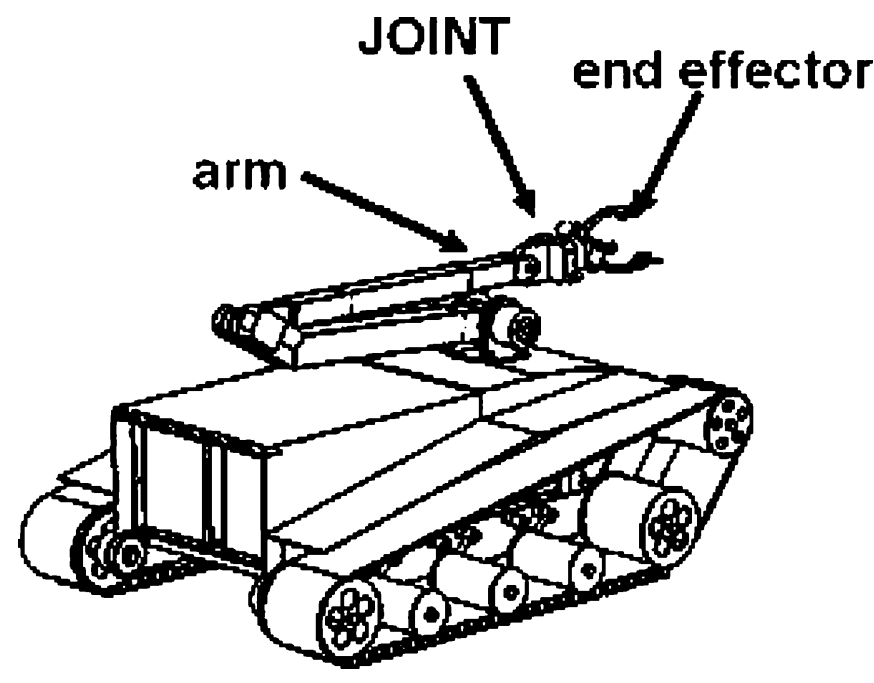

Fig. 1. The place of quick-changeable joint in the EOD robot.

This research consists of designing, analyzing, manufacturing, testing, and evaluating four different joints. In the design stage, some possible solutions for the joint are studied according to predefined design considerations. Four quick changeable joints with completely different working principles are designed. A test machine was built to test the life cycles of the joints. The critical parts of the joints and test machine are analyzed. These calculations are performed both in theory and in simulation programs.

After the theoretical design stage, the quick changeable joints and test machine are manufactured. The testing process is performed with real joints in the test machine to find their life cycles. Additionally, some other evaluating criteria such as weight, factor of safety, connection speed, number of components, connection simplicity, and reliability are considered in order to select the best joint design.

Finally, the conclusion of the test results is interpreted and the best design is determined of a completely mechanical quick changeable joint for multipurpose EOD robots. The future works describe the possible additional work for this research in order to increase the joint's performance and usability.

\section{Mechanical Design of Joints}

With the achieved joint designs, the robot does not require an extra electrical actuator to perform the tool changing process. Thus, the magnetic field production on the arm remains constant at low levels. Consequently, we get the new approach with various benefits which would increase the neutralizing capacity of EOD robots, and they are listed as follows:

1. Making the robot capable of automatic tool changing

2. Not making the robot bigger and heavier

3. Minimizing the magnetic field on the gripper

4. Eliminating the risk of using high-pressure systems on the robot

5. Not requiring the use of a pump, a tank, or valves of fluid systems

6. Simplifying the wiring of the tools

Different design constraints are defined as below for the quick changeable and automatic joint designs:

1. Maximum Load: The robot arm is expected to carry a maximum $10-\mathrm{kg}$ load. The connection must withstand the bending movement caused by a $10-\mathrm{kg}$ load.

2. Maximum Pull or Push: The goal for the arm is to pull or push a $100-\mathrm{kg}$ load without lifting the load. Different friction coefficients must be considered for different surfaces and the joint must withstand the tensile stress caused by the friction of 100-kg load.

3. Autonomy: The joint design must be able to provide an autonomous tool changing process.
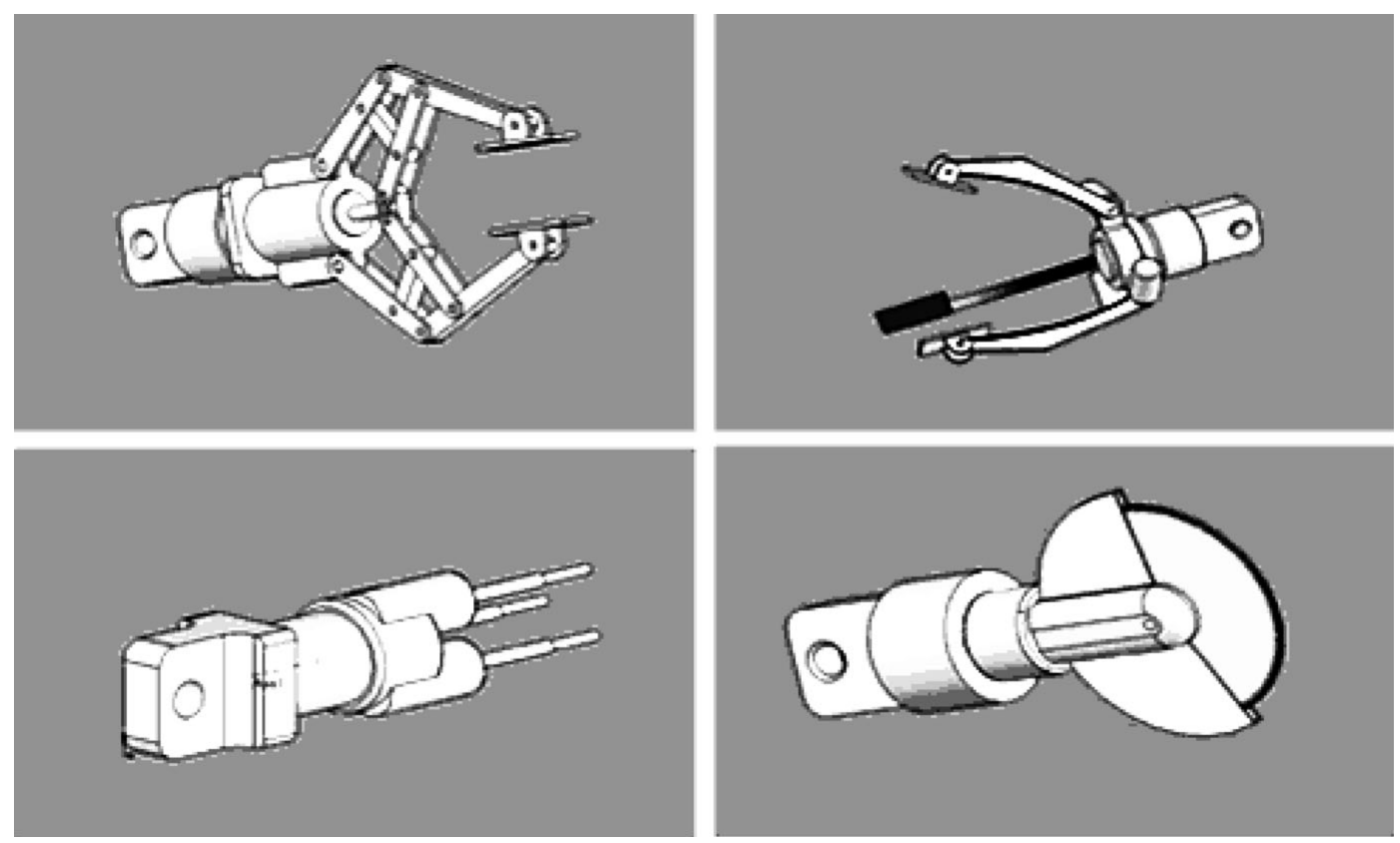

Fig. 2. Different-purpose tools of EOD robot. 

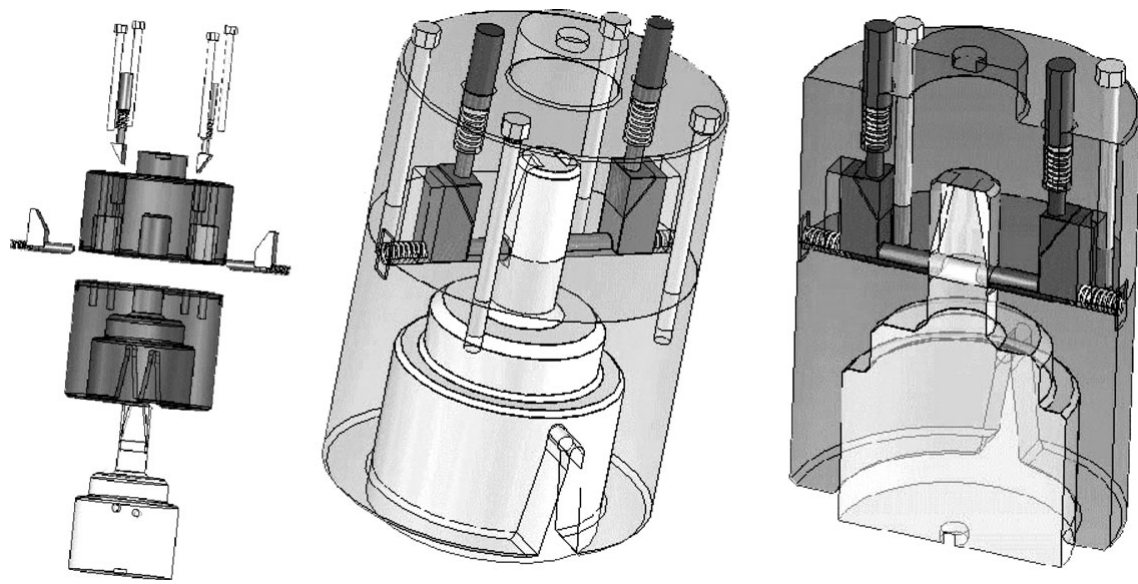

Fig. 3. The internal, exploded, and section views of the first joint.

4. Completely Mechanical: No extra actuator for the connection mechanism is allowed.

5. Connection Procedure: Only the motion of the arm is allowed for the activation and deactivation of the quick change connection mechanism.

6. Transmission: The joint must allow the transmission of load, torque, control signals, and electricity from the arm to the gripper.

7. Weight: The joint should be at an optimum weight which is less than $2 \mathrm{~kg}$ where it has enough mechanical strength required by constraints 1 and 2 .

\subsection{First joint design}

Basically, the first design is based on a simple slider mechanism. This design requires an arm motion with 2-DOF. In other words, in order to lock and unlock this joint, the robot arm must be able to move in two different axes.

This joint design is a direction-dependent design which means that there is only one way of locking male part and female part. The male part can enter the female part only at two symmetric positions.

The internal design and components of the joint are illustrated in Fig. 3. There are two main parts which are the male part and the female part. The female part consists of two pieces and contains two horizontal and two vertical slider pins and springs. The pins are grooved in an upper side of the female part and they can move inside this groove.

The working principle of joint 1 is illustrated in Fig. 4 which shows two discrete positions: the figure on the left side shows the locked position and that on the right shows the unlocked position of the joint. In the figure with the locked position, when the vertical forces are applied on top of the pins, vertical sliders start sliding down and make the horizontal sliders move left and right. The horizontal pins come out of the locking hole of the male part, causing the male part to separate from the female part. Locking pins are carried back by springs.

The lateral triangular-shaped slots in the bottom female part of the design let the male part enter the female part at the right position with a $20^{\circ}$ angle of tolerance.

Figure 5 shows the connection and disconnection probreak cesses of the joint design. In order to hold the gripper, the arm comes on top of the gripper and moves downward
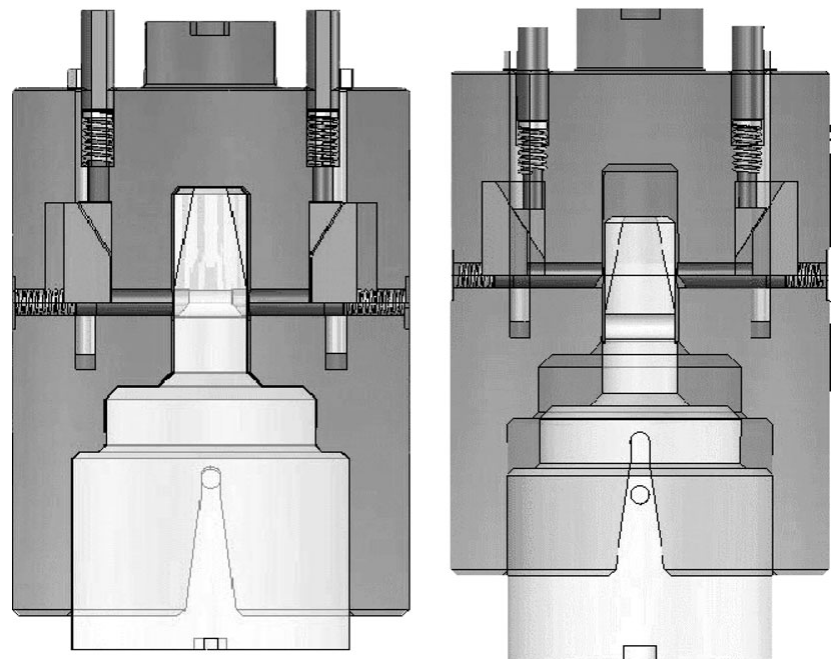

Fig. 4. Locked and unlocked position of the first joint.

until the male part is completely taken into the female part: the locking mechanism is activated and it is locked.

To release the gripper, the arm pulls the female part against the holder. In this way, the vertical pins are pushed and the horizontal pins release the male part equipped with the end effector.

\subsection{Second joint design}

The theory behind this joint design is, basically, based on the disk brake mechanism of automobiles. It requires 2-DOF motion in order to lock and unlock itself. Only a vertical motion is sufficient for locking; however, both horizontal and vertical motions are required for unlocking.

The connection of a second joint is achieved without any direction constraints which means the male part has no entrance angle and position because of its $360^{\circ}$ symmetric design.

The female part consists of two pieces which are called top and bottom pieces. The bottom piece has special grooves for the elements of the locking mechanism. These elements are one horizontal C-shaped segment, one activation button, two pins, and a spring.

The working principle of the second joint is mainly the same as the first joint. The $\mathrm{C}$-shaped segment in the female 

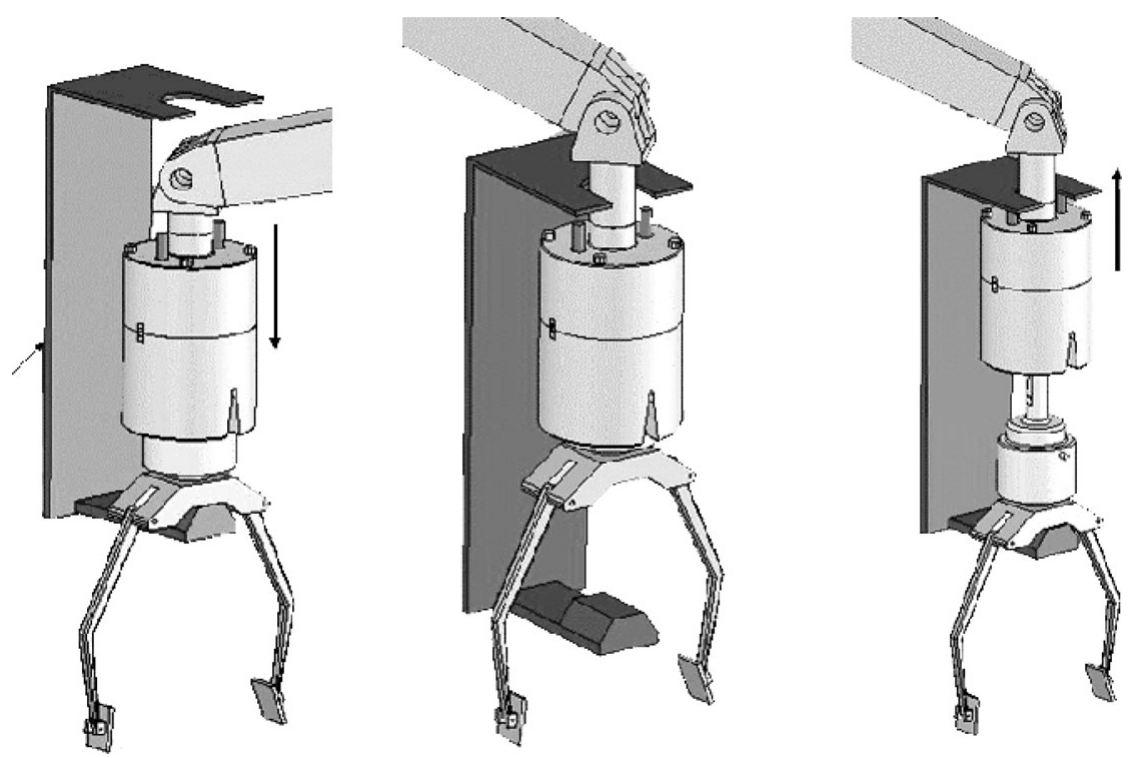

Fig. 5. Connection steps of the first joint.
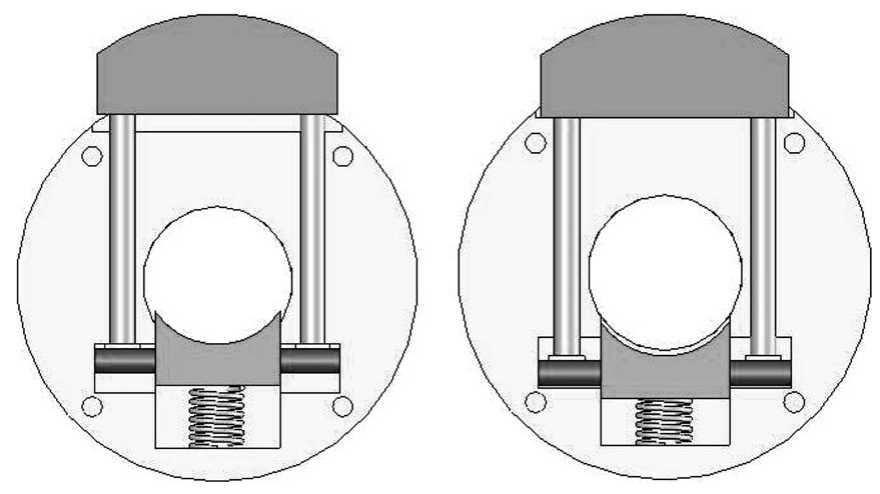

Fig. 6. Locked and unlocked position of the second joint.

part enters the male part which enables the locking of male and female parts.

The unlocking process requires pushing the lateral button along to the center of the joint. By this way, the locking segment comes out from the slot of the male part and causes the joint to unlock, Fig. 6.

The connection process is very simple for this design; the arm equipped with the female part comes on top of the male part which is attached to the gripper and by moving downward the joint becomes locked.

When the robot needs to change its gripper, it should make a horizontal motion in order to push the button against the wall of the tool holder on the robot's body causing the male part with the gripper to detach. The disconnection procedure is illustrated in Fig. 7.

\subsection{Third joint design}

The working principle behind this joint design comes from a locking mechanism of a door. As it is the same as with the door locks, the female part of the joint must be able to turn with a $90^{\circ}$ angle in order to provide connection. A vertical motion and a rotational motion are required for this joint.

This joint consists of a few basic parts which are easy to manufacture and assemble. Figure 8 shows the components
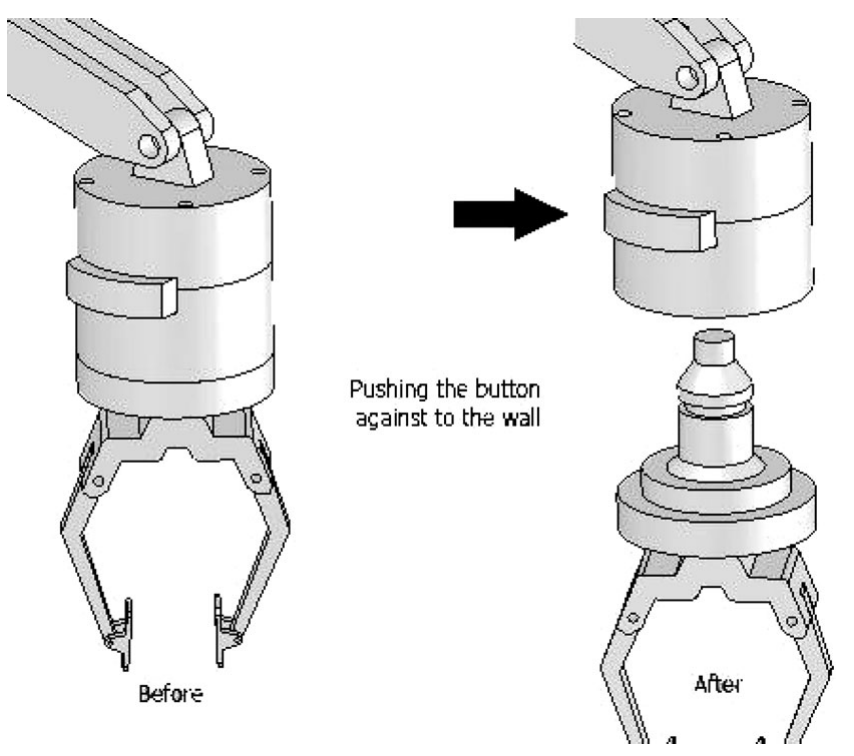

Fig. 7. Disconnection procedure of the second joint.
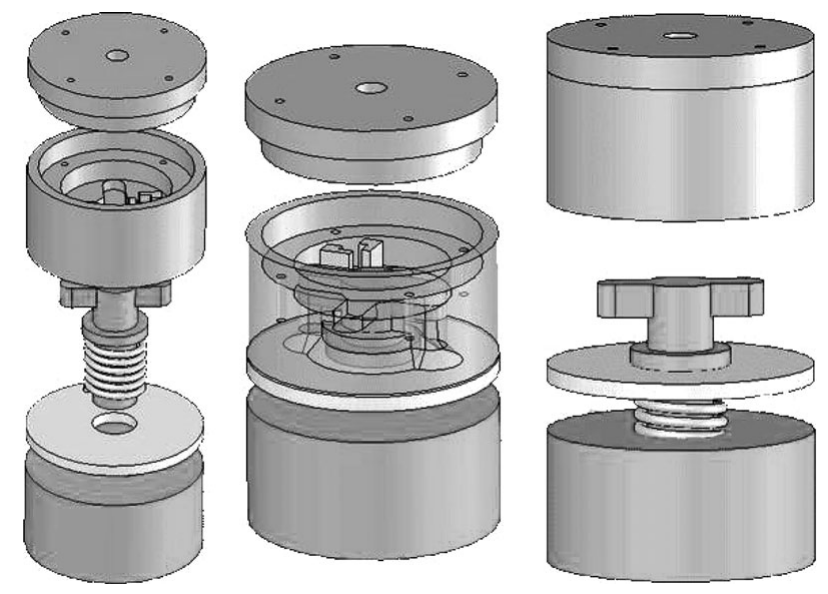

Fig. 8. Exploded, internal, and general views of the third joint. 

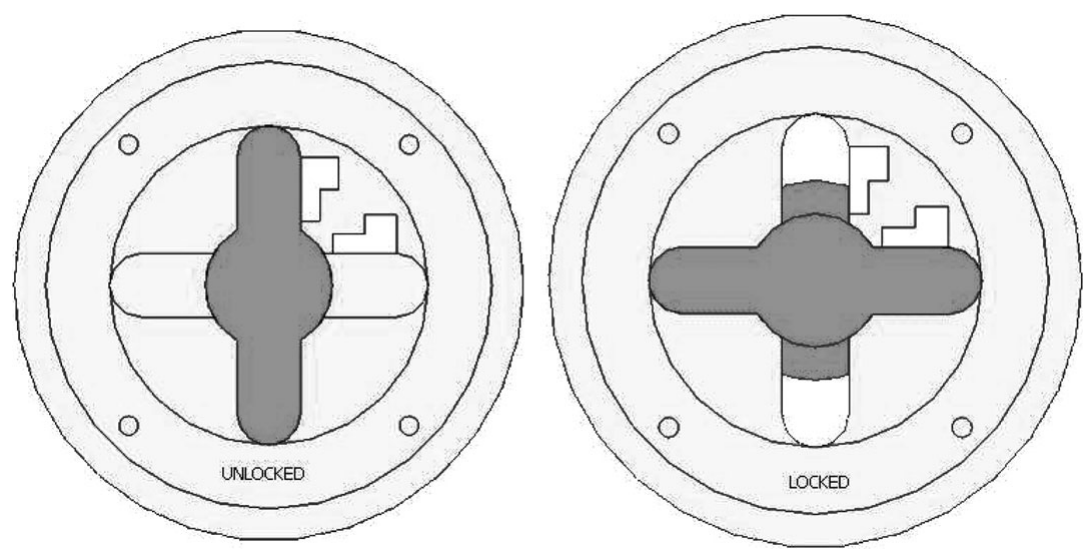

Fig. 9. Locked and unlocked positions of the third joint.

of the joint. In the male part there is one T-shaped locker element and there is a ring around the T-shaped part which is supported by the spring.

The T-shaped locker part enters the hole of the female part and the female part turns $90^{\circ}$ with the help of the robot arm. When the turning process is completed, the joint locks itself automatically as shown in Fig. 9. In the locked position of joint, it is not possible to turn the male part in the female part.

The arm comes on top of the griper and by moving down, the male part enters the female part. The arm applies vertical force to compress the spring in the joint, until the top of the male part hits the inner surface of the female part. To activate the locking mechanism, the female part must turn $90^{\circ}$ clockwise with compressive force. In order to unlock the joint, the female part should be pushed down and rotated counterclockwise. Figure 10 shows the disconnection procedure.
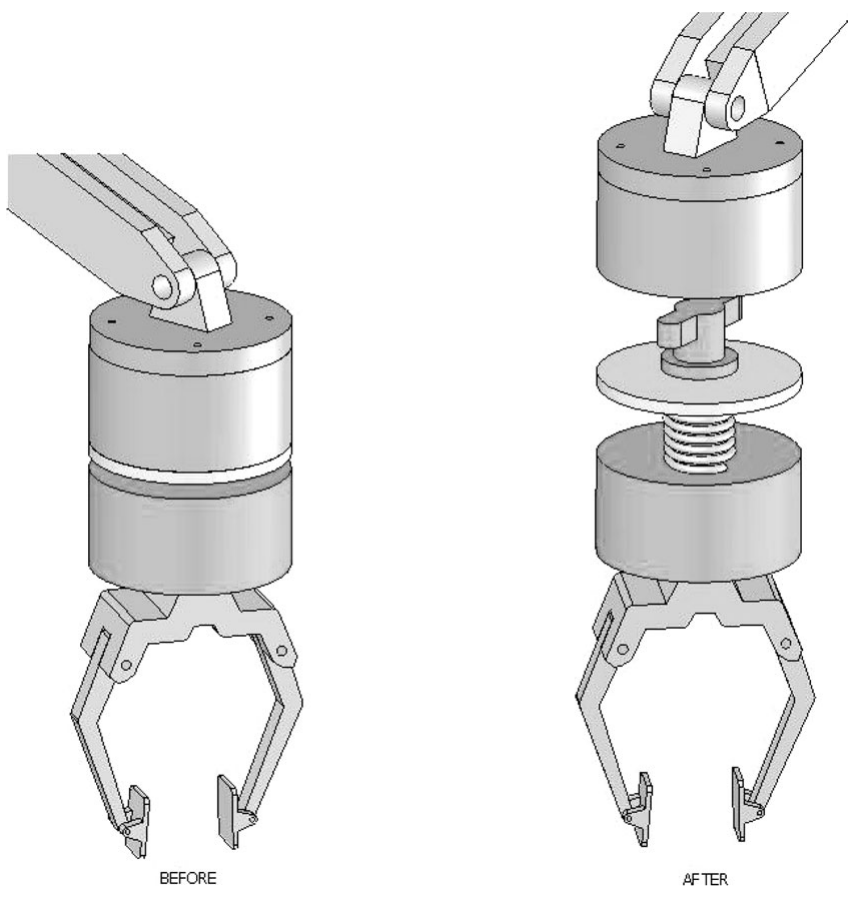

Fig. 10. Disconnection procedure of the third joint.

\subsection{Fourth joint design}

The idea behind this joint is similar to the switch mechanism of pens. There are two positions on its cam mechanism. One position of the cam is used for unlocking the joint while the other position is used for locking the joint. Therefore, one motion is used for both locking and unlocking of the joint which means it requires only 1-DOF motion of the robot arm.

As it is seen in Fig. 11, this design has more parts than the others. It is similar to the other female parts since it consists of two portions and some grooves for the latching elements; however, the male part design is much simpler than that of other joints. In the upper portion of the female part, there are three grooves located symmetrically at $120^{\circ}$ angle. The lock mechanism consists of a gear, one unidirectional bearing, one ball, and one cam in each groove. To activate gear mechanisms, there are three identical worm geared pins and springs and a support wristlet for the pins.

When the geared pin is pushed, the pin forces the gear to turn around a half cycle (Fig. 12). The bearing located at the center of the gear is locked and rotates both the shaft and the cam attached to the shaft. The cam is turned from the lower position to the upper position and this causes the ball to come out its hole (Fig. 13). As a result, the male part is locked in the female part. So, the force exerted by the robot to the pins can be stopped. When the geared pin is pushed back with the help of the spring, the gear turns in a reverse
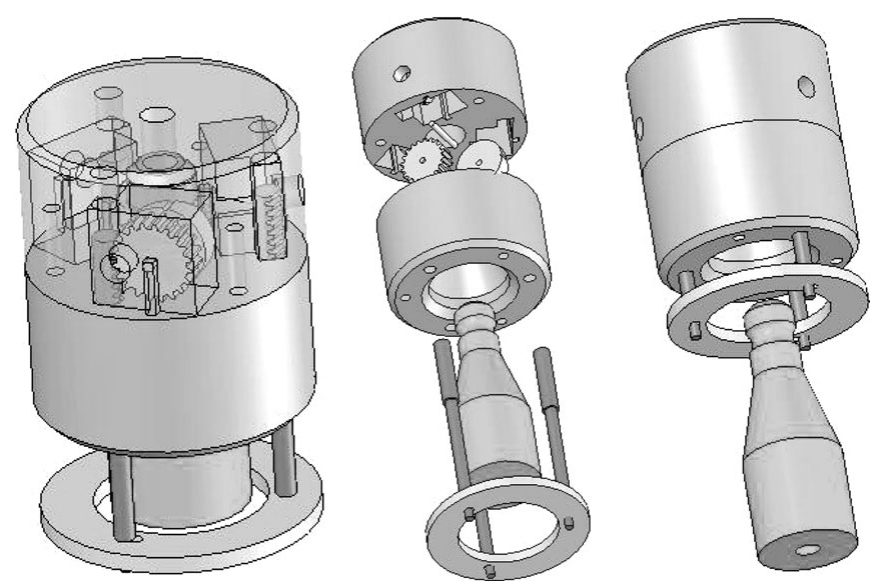

Fig. 11. Exploded, internal, and general views of the fourth joint. 

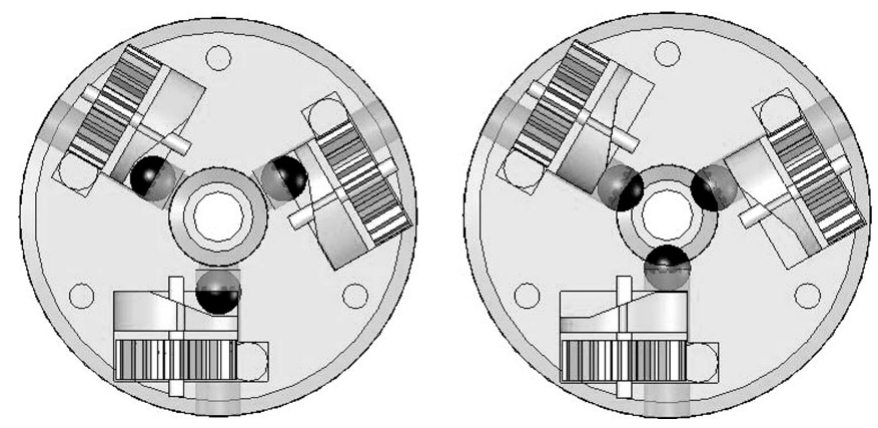

Fig. 12. Rocker pin and cam before and after pushing.
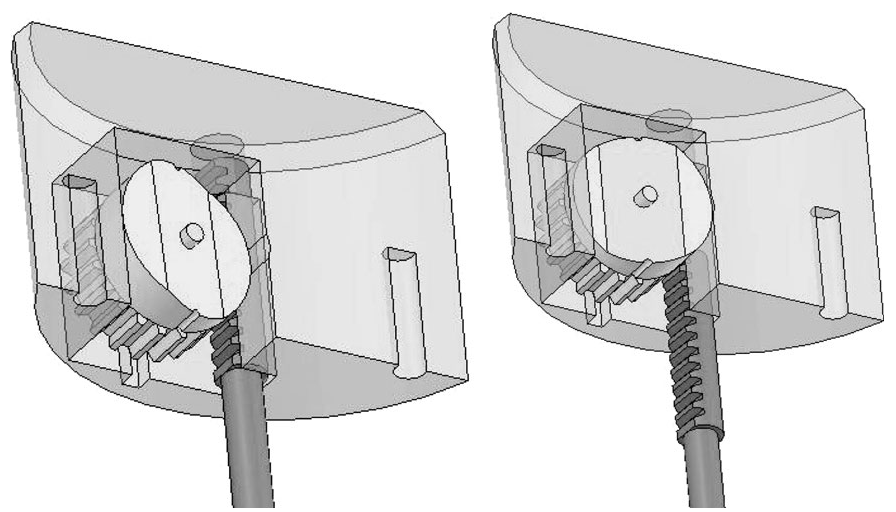

Fig. 13. Locked and unlocked positions of the fourth joint.

direction while the cam does not rotate. To unlock the joint the same operation must be done.

The robot arm comes on top of the gripper located in the magazine of the robot body and is moved closer to the gripper till the bracket contacts the magazine (Fig. 14). Then it pushes the female part of the joint against the magazine since the pins of the bracket come completely into the female part, so the male part is locked. In order to release the gripper, the robot needs to do the same procedure for locking the gripper. This joint is different from all other joints since it only requires one vertical motion to lock and unlock the gripper to the arm.

\subsection{Control and power signal transmission}

Quick changeable joints should allow power and control signals to be passed to the gripper. In order to achieve power and control signal transfer, electrically conductive plates are placed on the inside of the female part as well as at the tip of the male part. These transmission plates are inside each other and are in ring shapes. Figure 15 shows the designed power signals' connection for the second joint design; however, they are not realized on the actual design.

\subsection{Materials selection}

In order to select the optimum material for quick changeable joints, mechanical and physical properties of materials are considered. These properties are tensile strength, yield strength, shear strength, density, and magnetic property, especially.

Because of its unique properties aluminum-T3 alloy is used in manufacturing four different quick changeable joints and the test machine. Moreover, the machining of aluminum-T3 is much easier and its density is much lower than other metals, which means the weights of the joints are as low as possible. However, some small parts, like horizontal slider pins in the first joint or the horizontal buffer in the second design are manufactured from Steel 302 because of the required high shear strength. Since these are very small parts, they only cause a negligible, almost $2 \%$, amount of weight increase.

\section{Test Machine Design}

The test machine is required for finding the quick changeable joints' life spans and reliabilities. The test arm on the machine can move upward and downward with pneumatic actuators. This machine achieves the motions of an EOD
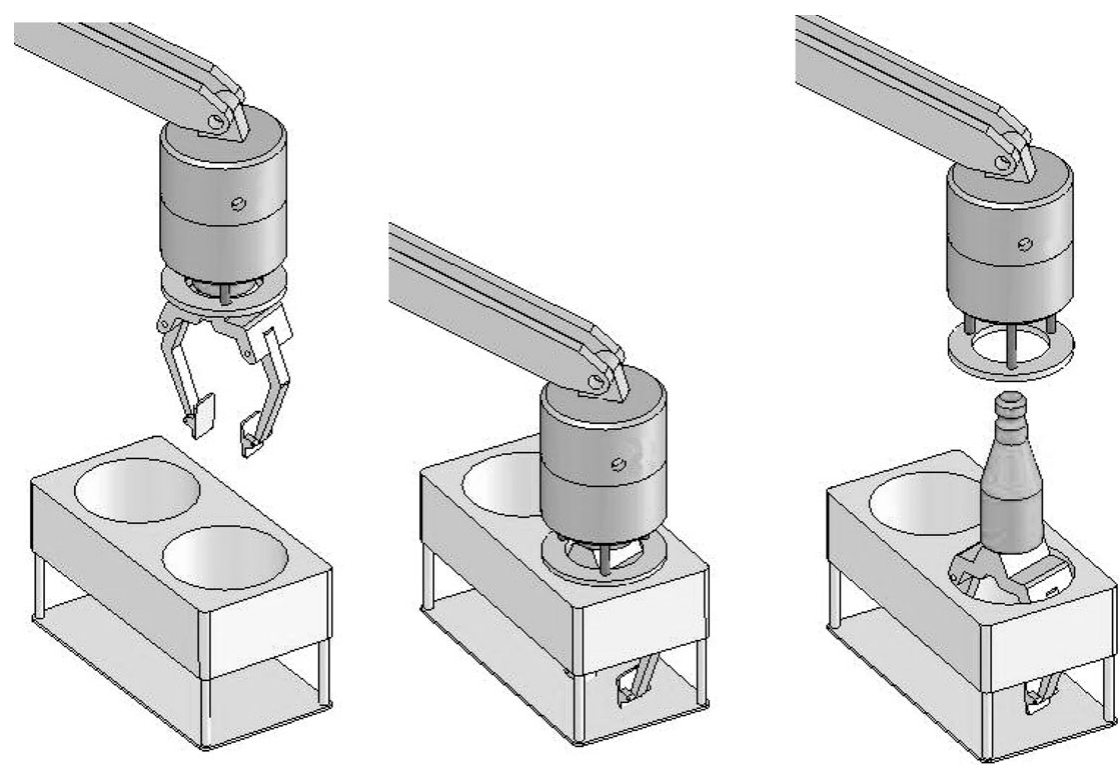

Fig. 14. Disconnection procedure of the fourth joint. 

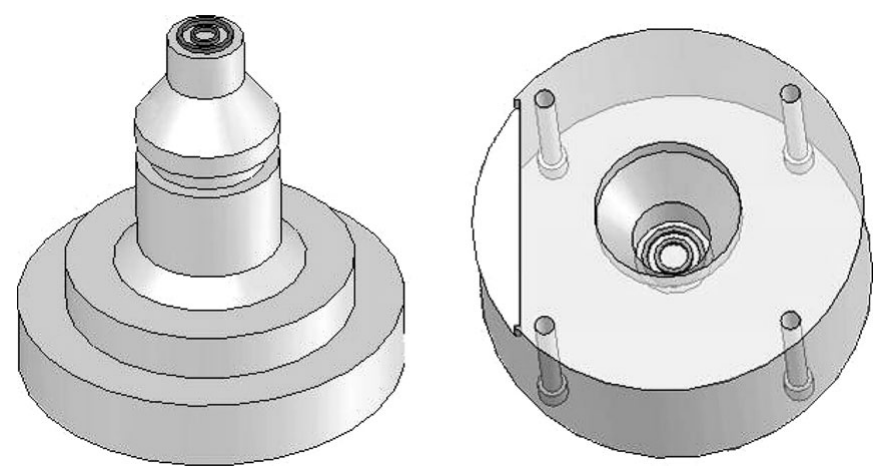

Fig. 15. Power transmission rings on the second joint.

robot arm which are necessary for locking and unlocking the quick changeable joints. While moving up and down, the test machine has some additional pneumatic actuators to do auxiliary motions which are required for the joint disconnection, such as turning of a quarter revolution and horizontal displacement.

In this paper, the mechanical design of the test machine including its working principle, machine parts, and testing procedure is examined. The electronic control system and its components are also explained.

\subsection{Mechanical system design}

The mechanical parts of the test machine are designed in order to meet the requirements of quick changeable joints. All joints need to be lifted in a vertical direction where joints 2 and 3 need an extra motion to activate their unlocking mechanisms. Figure 16 shows the test machine made of iron with a vertical lifting mechanism and one horizontal triggering mechanism. This lifting mechanism moves along a vertical path and provides joint connection by lifting the female part where the male part is attached to the vertical pin located at the bottom of the table. The horizontal arm located

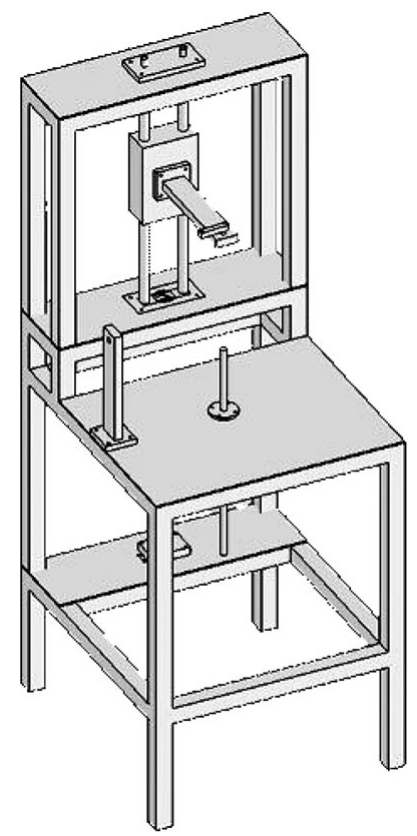

Fig. 16. 3D design view of the test machine.

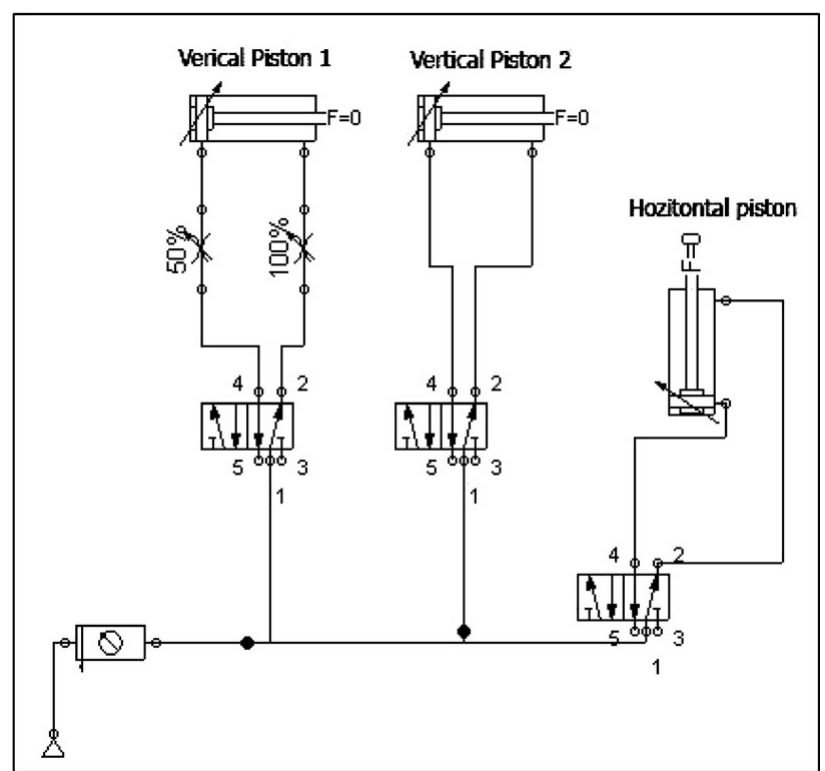

Fig. 17. Pneumatic system circuit of the test machine.

on the side of the table is used to attach a cylinder to make a horizontal pusher for the second joint design. Due to the simple design of the test machine, it was easy to manufacture.

\subsection{Pneumatic system design}

As an actuation system, pneumatics is preferred in the testing machine because of its high speed and ease of use. The test machine has one testing arm and the female part of a quick changeable joint is attached to it. The male part of the joint is fastened to the bottom surface of the testing machine. Two cylinders are used to create the vertical motion of the arm so that there is a three-position motion. There is also another short stroke cylinder for the horizontal motion in order to push the button of the second joint and to turn the female part at a $90^{\circ}$ angle of the third joint enabling it to unlock. Figure 17 illustrates the basic circuit of the pneumatic system. The pneumatic components and the working principle of the components are explained in the next section.

The main linear actuators of the pneumatic systems are cylinders. There are many kinds of cylinders due to the diameters and the stroke lengths. The diameter determines the force exerted by a cylinder whereas the stroke determines the distance between two positions of the cylinder shaft. In this project, three cylinders with different strokes, 25, 100, and $200 \mathrm{~mm}$ all having a $25 \mathrm{~mm}$ diameter, are used.

The pneumatic cylinders are controlled by special-type valves. These valves drive the cylinders by canalizing the compressed air through the gates of the cylinders. Generally, valves have one air inlet, two cylinder outlets, and two outlets for the air exhaust. The gates connected to the cylinders are named as A and B ports. The exhaust ports are R and S outlets, and the $\mathrm{P}$ port is the compressed air inlet. When the valve is activated, it connects both the compressed air inlet P to the outlet B and the exhaust air R to the outlet A, so the shaft of the cylinder changes its position. The activation of pneumatic valves is achieved with electricity.

The air source treatment unit includes a filter, a regulator, a filter regulator, and a lubricator. The lubricator is an element 


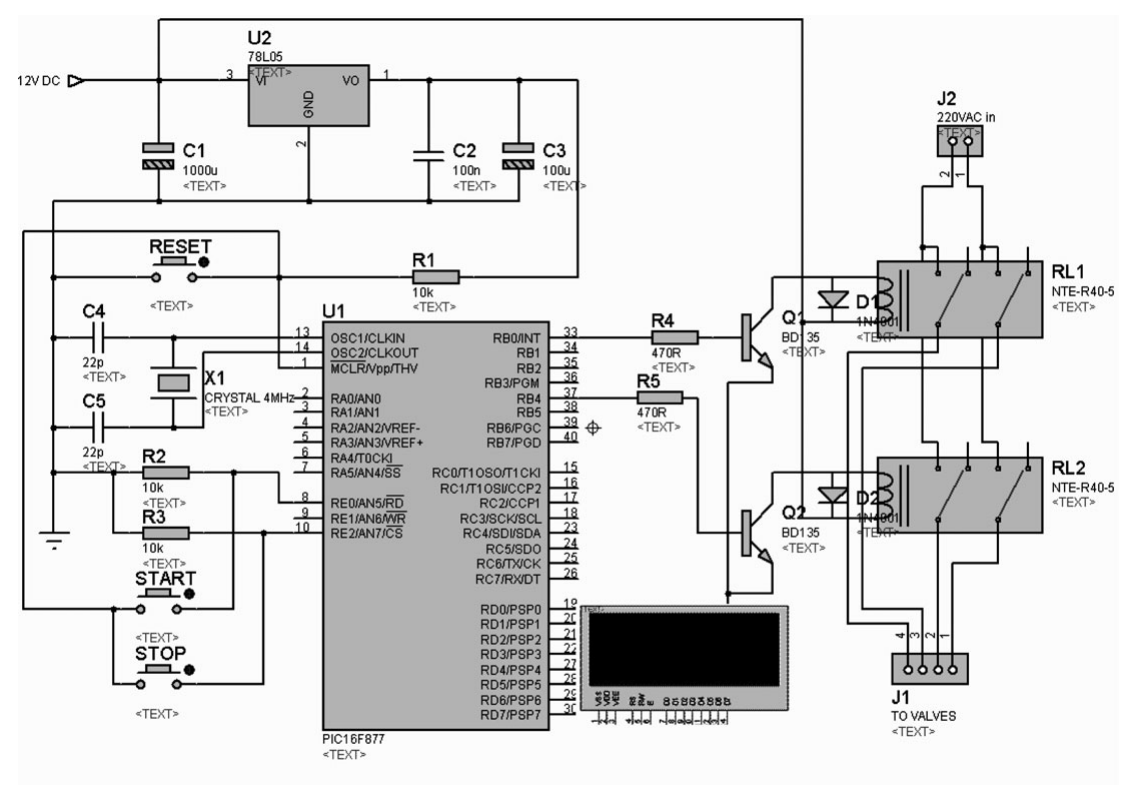

Pneumatic test machine controller circuit scheme

Fig. 18. Circuit scheme of the test machine controller.

which provides required lubrication for the pneumatic system via an oil drip that can be easily adjusted as needed.

\subsection{Electronic system design}

The main goal of an electronic circuit is to control the pneumatic valves and provide motion of the test arm in order to connect and disconnect the joints. While the joint is being tested, a trial number is counted by a micro-switch controlled by the electronic circuit.

A special control circuit is required for the test machine to control the pneumatic valves. For the four different joint designs, there are four different connection processes. For example, the first joint design only requires an upward and downward motion while the second joint design requires both, vertical and horizontal motions. Because of this fact, an electronic controller is needed to control different valves in different sequences for each joint design.

Because of its simplicity and reliability, the PIC16F877 is used in the control circuit. This microcontroller runs at speed up to $20 \mathrm{MHz}$. It has $33 \mathrm{I} / \mathrm{O}$ pins which are grouped under four main ports called Port A, B, C, and D. These ports can be configured as input or output as required by the task.

The electronic components in the circuit run with the voltage of $5 \mathrm{~V}$, so the $12 \mathrm{~V}$ DC voltage supplied by battery is reduced to the $5 \mathrm{~V} \mathrm{DC}$ with 7805 voltage regulator, and it is filtered by the high-pass and low-pass capacitors $\mathrm{C} 2$ and $\mathrm{C} 3$ (Figs. 18 and 19).

PIC16F877 runs at $5 \mathrm{~V} \mathrm{DC}$ and at the speed of $4 \mathrm{MHz}$ which is determined by the oscillator crystal. Two parallel $22 \mathrm{pF}$ capacitors are used for eliminating the noises which affect the oscillator in the circuit. Two double contact relays of $12 \mathrm{~V}$ nominal voltage are triggered by BD135 transistors and protected by $1 \mathrm{~N} 4001$ diodes. These relays switch the pneumatic valves connected to terminal $\mathrm{J} 1$ and $\mathrm{J} 2$ with the input voltage of $220 \mathrm{~V}$ AC.

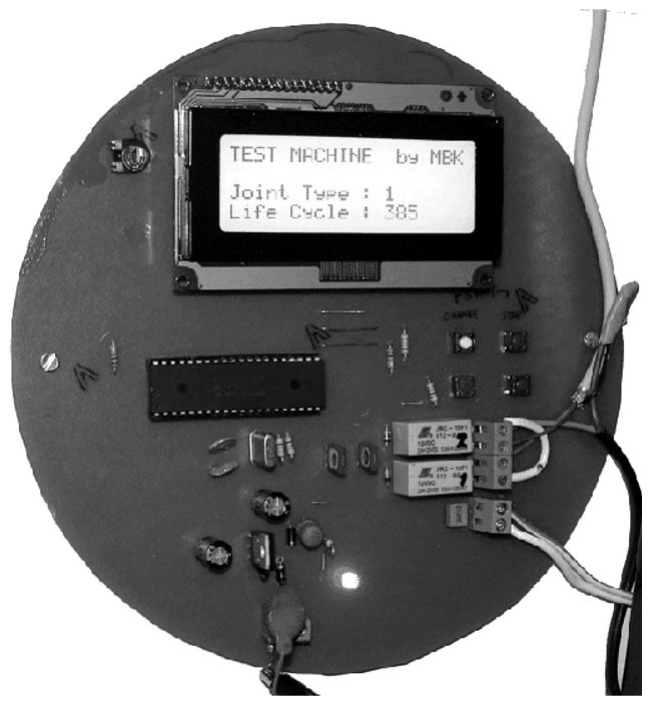

Fig. 19. Finished view of the test machine controller circuit.

A four-line Hitachi compatible LCD is used in the circuit for showing the number of connections of each joint. The LCD uses parallel communication via Port D of PIC16F877.

Three push buttons were used in order to collect input parameters such as joint type, start or stop command for the test process, and resetting the system. A micro-switch located under the male part of the joint is connected to the input port of the PIC to make an input signal when the joint connects. The program codes for PIC 16F877 is written in PIC Basic.

Manufacturing of the test machine is achieved in two stages. The first stage is the manufacturing of the vertical lifting mechanism and the second stage is the manufacturing of the iron test table.

In the first stage, a test arm and its vertical slider mechanism are produced from aluminum-T3. The test arm 


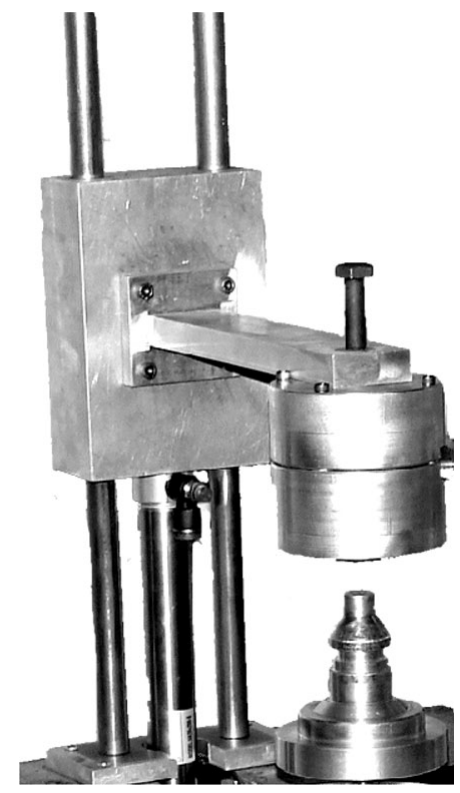

Fig. 20. Vertical slider mechanism of the test machine.

is milled from one piece. When it is considered that this part is used for lifting and pushing the joints, bending and shear forces occur. Because of this fact, the test arm is produced as one piece. Figure 20 shows the manufactured vertical slider mechanism.

In the second stage, the test table is constructed by welding iron bars. Figure 21 illustrates the complete test machine.

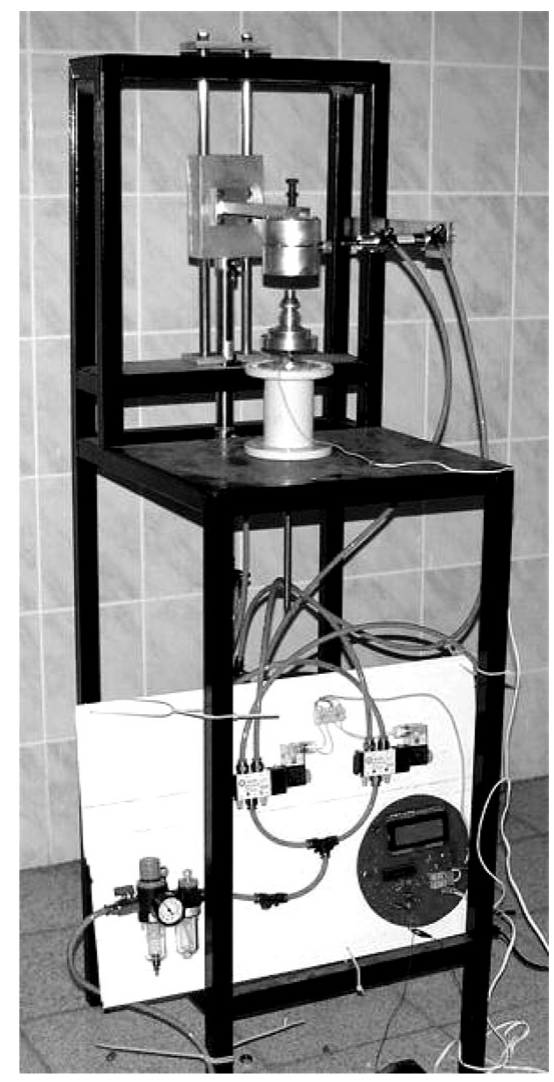

Fig. 21. Life cycle test machine.

\subsection{Strength analysis of the test machine}

During the testing process, the arm-the critical element of the test machine - needs to push and pull the joint to lock and unlock it, causing bending stress. A vertical force applied by the arm is $50 \mathrm{~N}$ which is enough for the locking mechanism. The cross-sectional area is $600 \mathrm{~mm}^{2}$ and the distance between the force and the fixed point is $185 \mathrm{~mm}$.

The deflection $\Delta y$ is calculated as $0.548 \times 10^{-6} \mathrm{~m}$ for the arm part which is negligible where the shear modulus of aluminum-T3 alloy is $28 \mathrm{GPa}$.

Considering the results from the factor of safety and deflection calculations, it is understood that there is no problem in strength of material on the critical part of the test machine.

\section{Testing of Joints}

After the joints are manufactured (Fig. 22), all joints are measured for their physical properties. These properties are summarized in Table I.

As it is seen from this table, all the joints are at almost the same weight and size. However, the number of components changes drastically between the second and fourth joints. While the required DOF of the arm is low at joint 4 the complexity of the mechanism is higher than the other joints. It is this complexity that makes this joint easier to operate; however, it also increases the risk of failure.

In the testing procedure, the joints were attached to the test machine and they were connected and disconnected 1000 times. These tests prove that the joint mechanisms work. During these tests, the connection time for each joint was also measured (Table I).

When the different failure modes are considered, the possible results of a failure is identified as jamming, breaking, corrosion, and fatigue.

Jamming can be a result of the moving parts rusting or dust filling into the gaps. In order to prevent these failures the moving parts of the joints should be manufactured of stainless materials. To avoid dust filling the gaps of the joints, the joints can be covered or sealed.

Breaking, chipping as well as corrosion will cause the joints to vibrate because of the increase of the gaps. Making

Table I. Properties of the designed and manufactured quick changeable joints.

\begin{tabular}{lcrrrr}
\hline Property & Importance & Joint 1 & Joint 2 & Joint 3 & Joint 4 \\
\hline 1 Weight (g) & 10 & 9 & 9 & 10 & 9 \\
2 Connection & 10 & 10 & 7 & 5 & 5 \\
time (s) & 30 & 18 & 28 & 30 & 10 \\
$\begin{array}{c}\text { Number of } \\
\text { components }\end{array}$ & 20 & 15 & 20 & 20 & 10 \\
$4 \begin{array}{c}\text { Number of } \\
\text { springs }\end{array}$ & 10 & 10 & 10 & 10 & 20 \\
$5 \begin{array}{c}\text { Required DOF } \\
\text { of arm }\end{array}$ & 100 & 72 & 84 & 75 & 64 \\
\hline $\begin{array}{c}\text { Holding during } \\
\text { disconnect }\end{array}$ & 10 & 10 & 0 & 10 \\
\hline Total & & 10 & & & \\
\hline
\end{tabular}



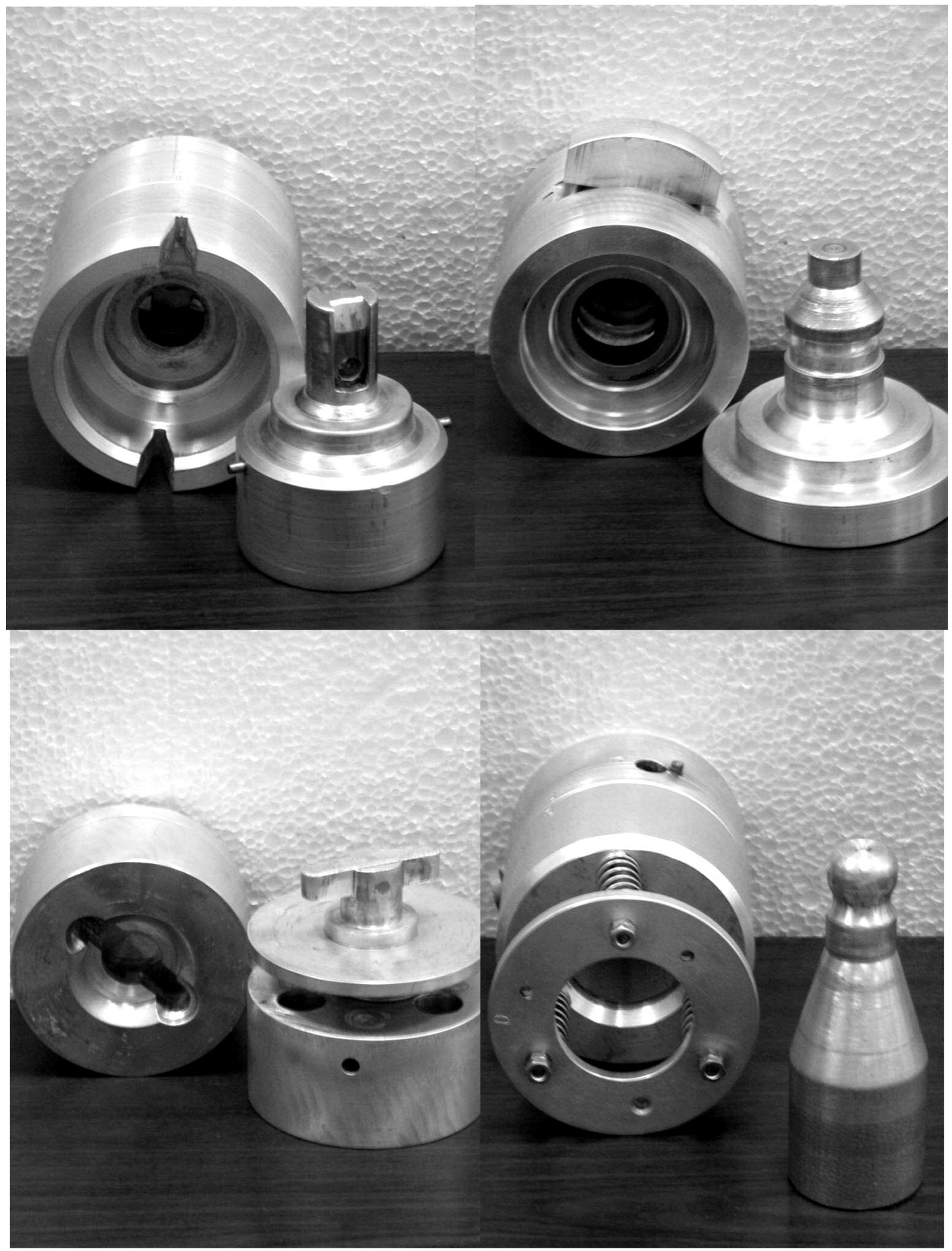

Fig. 22. Male and female parts of the four manufactured joints.

the joints from harder material or applying heat treatment on the critical parts will decrease the possibility of these failures to occur. Thermal or chemical corrosion will not occur, since the joints are not used in harsh environments. However, if this was the case, the environmental effects should be taken into account in the selection of the materials.

Fatigue occurs on the parts which are under cyclic load. In these joint designs, both the latches and the springs are forced with cyclic load. However; considering that the loading is under $3 \mathrm{MPa}$, the fatigue can happen after $10^{8}$ loading, which is acceptable for the application.
During the testing of the joints, jamming, breaking, chipping, visible corrosion, or fatigue is not observed.

For an EOD operation, the factor of parameters and points each joint received is shown in Table II.

According to these test results, the second joint is selected as the best design.

\section{Conclusion and Future Works}

In conclusion, this project shows how a quick changeable joint is a necessary part of an end effector changer where 
Table II. Importance of the parameters and joints' points.

\begin{tabular}{lcrrrc}
\hline Property & Importance & Joint 1 & Joint 2 & Joint 3 & Joint 4 \\
\hline 1 Weight (g) & 10 & 9 & 9 & 10 & 9 \\
2 Connection & 10 & 10 & 7 & 5 & 5 \\
$\begin{array}{c}\text { time (s) } \\
3 \text { Number of } \\
\text { components }\end{array}$ & 30 & 18 & 28 & 30 & 10 \\
$4 \begin{array}{c}\text { Number of } \\
\text { springs }\end{array}$ & 20 & 15 & 20 & 20 & 10 \\
$5 \begin{array}{c}\text { Required DOF } \\
\text { of arm }\end{array}$ & 20 & 10 & 10 & 10 & 20 \\
$6 \begin{array}{c}\text { Holding during } \\
\text { disconnect }\end{array}$ & 10 & 10 & 10 & 0 & 10 \\
Total & 72 & 84 & 75 & 64 \\
\hline
\end{tabular}

more than one job is being performed by the EOD robot and all of the necessary end effectors cannot be assembled to the robot arm. A completely mechanical quick changeable joint can achieve the end effector changing process efficiently and effectively requiring only basic motions of the robot arm.

In this study, four unique quick changeable joints are designed and manufactured. Reliability and life cycle tests are done with these joints by using a manufactured test machine. In the evaluation part, it is proven that each joint has some properties better than the others. For example, by the properties of $360^{\circ}$ symmetric design and nonrotating male part design, joint 1 is more suitable as a tool holder for CNC machines where joint 3 is more appropriate for the practical connection of vehicle pulling and load lifting applications because of its high factor of safety. Joint 4 is better if there is a limitation on DOF of the robot arm. However, when all of the parameters are considered, the joint which satisfies all parameters together must be chosen as the best quickchangeable joint for the EOD robot, which is joint 2.
In future work, some modifications on these joints can be done to enhance their performance and as a result the usage of quick changeable joints can be extended into many different areas such as lifting and pulling applications and different tool changing applications. In the critical parts of the joints, different steel alloys can be used instead of aluminum alloy and this will provide improved lifting capacity of the joint.

\section{Acknowledgments}

The author would like to thank Mr. Bahattin Kor for his help in the manufacturing and the testing stages of the joints. This project is financially supported by the State Planning Agency of Turkey under the contract number of 2005DPT13.

\section{References}

1. Telerob web site, July 31, 2008, http://www.telerob.de/telerob_ online/index.html

2. Allen Vanguard web site, July 31, 2008, http://www. allenvanguard.com

3. M. I. Gokler and M. B. Koc, "Design of an automatic tool changer with disc magazine for a CNC horizontal machining center," Int. J. Mach. Tools Manufact. 37(3), 277-286 (1997).

4. B. Ryuh, S. M. Park and G. R. Pennock, "An automatic tool changer and integrated software for a robotic die polishing station," Mech. Mach. Theory 41(4), 415-432 (2006).

5. A. Meghdari and F. Barazandeh, "Design and fabrication of a novel quick-change system," Mechatronics 10(7), 809-818 (2000).

6. S. Hesse, "Modular Pick and Place Devices," In: Blue Digest on Automation (Festo Ag\&Co., Esslingen, Germany, 2000).

7. S. Hesse, "Grippers and Their Applications," In: Blue Digest on Automation (Festo Ag\&Co., Esslingen, Germany, 2001).

8. M. S. Rosheim, "Robot Wrist Actuators (John Wiley \& Sons, Canada, 1989). 
Reproduced with permission of the copyright owner. Further reproduction prohibited without permission. 